\title{
Unravelling the enzymatic degradation mechanism of supramolecular peptide nanofibers and its correlation with their internal viscosity
}

\author{
Yejiao Shi, Peter A Summers, Marina K. Kuimova, and Helena S. Azevedo
}

Nano Lett., Just Accepted Manuscript • DOI: 10.1021/acs.nanolett.0c02781 • Publication Date (Web): 31 Aug 2020

Downloaded from pubs.acs.org on September 1, 2020

\section{Just Accepted}

"Just Accepted" manuscripts have been peer-reviewed and accepted for publication. They are posted online prior to technical editing, formatting for publication and author proofing. The American Chemical Society provides "Just Accepted" as a service to the research community to expedite the dissemination of scientific material as soon as possible after acceptance. "Just Accepted" manuscripts appear in full in PDF format accompanied by an HTML abstract. "Just Accepted" manuscripts have been fully peer reviewed, but should not be considered the official version of record. They are citable by the Digital Object Identifier (DOI®). "Just Accepted" is an optional service offered to authors. Therefore, the "Just Accepted" Web site may not include all articles that will be published in the journal. After a manuscript is technically edited and formatted, it will be removed from the "Just Accepted" Web site and published as an ASAP article. Note that technical editing may introduce minor changes to the manuscript text and/or graphics which could affect content, and all legal disclaimers and ethical guidelines that apply to the journal pertain. ACS cannot be held responsible for errors or consequences arising from the use of information contained in these "Just Accepted" manuscripts. 


\title{
Unravelling the enzymatic degradation mechanism of supramolecular peptide nanofibers and its correlation
}

\section{with their internal viscosity}

Yejiao Shi, ${ }^{1 *}$ Peter A. Summers, ${ }^{2 *}$ Marina K. Kuimova, ${ }^{2 *}$ and Helena S. Azevedo ${ }^{1 *}$

${ }^{1}$ School of Engineering and Materials Science \& Institute of Bioengineering, Queen Mary, University of London, Mile End Road, London, E1 4NS, UK

${ }^{2}$ Department of Chemistry \& Molecular Science Research Hub, Imperial College London, White City Campus, London, W12 0BZ, UK

KEYWORDS: peptide nanofibers, cathepsin B, enzyme degradation, internal viscosity, molecular rotors, fluorescence lifetime imaging

\begin{abstract}
:
Enzyme-responsive supramolecular peptide biomaterials have attracted growing interest for disease diagnostics and treatments. However, it remains unclear whether enzymes target the peptide assemblies or dissociated peptide monomers. To gain further insight into the degradation
\end{abstract}


mechanism of supramolecular peptide amphiphile (PA) nanofibers, cathepsin B, with both exopeptidase and endopeptidase activities, was exploited here for degradation studies. Hydrolysis was found to occur directly on the PA nanofibers as only surface amino acid residues were cleaved. The number of cleaved residues and the degradation efficiency were seen to be negatively correlated with the internal viscosity of the PA nanofibers, quantified to be between $200-800 \mathrm{cP}$ (liquid phase) using fluorescence lifetime imaging microscopy combined with an environmentally sensitive molecular rotor, BODIPY-C10. These findings enhance our understanding on the enzymatic degradation of supramolecular PA nanofibers and have important implications for the development of PA probes for the real-time monitoring of disease-related enzymes. 
Inspired by the numerous enzyme regulated processes in living organisms, enzyme-responsive biomaterials have been designed to exert their functions specifically and selectively in response to the abnormal expression of enzymes at disease sites. ${ }^{1}$ As such, they are particularly attractive for disease diagnostics and treatments. ${ }^{2,3}$ One popular approach to fabricate enzyme-responsive biomaterials is supramolecular self-assembly, in which the enzyme-sensitive functionalities, typically peptide sequences, can be rationally incorporated into self-assembling units able to form various types of nanostructures. ${ }^{4-7}$ Responding to the enzymatic activity, these supramolecular systems can undergo different pathways, including molecular assembly, ${ }^{8-10}$ disassembly, ${ }^{11}$ and also dynamic molecular reconfigurations. ${ }^{12-15}$ These processes can result in the formation, dissociation and transition of the assemblies, endowing them with desirable properties for diverse biomedical applications, ranging from selective cancer cell killing, ${ }^{16-18}$ targeted therapeutic release, ${ }^{19-21}$ to cell-mediated remodeling of extracellular matrix mimics. ${ }^{6,22}$

One important consideration when designing enzyme-responsive supramolecular biomaterials is the accessibility of the substrate to the enzyme. The cleavable site can be easily reached when the molecules are in the monomeric state (e.g. during enzyme-triggered assembly processes), but it can be limited, or even completely restricted, when the molecules are in the assembled state, especially in filaments (e.g. during enzyme-triggered disassembly and structure transition processes). Two main strategies have been pursued to address the lack of enzyme accessibility. One strategy is to expose the enzyme cleavable sequence on the surface of the assemblies, ${ }^{21}$ or incorporated in a cross-linker applied post-assembly, ${ }^{23}$ where the susceptible bond can be reached more easily by the enzyme. The other strategy is to incorporate the enzyme degradable sequence into self-assembled peptides with various backbone conformations, including $\beta$-sheet, ${ }^{4,}{ }^{6} \beta$ hairpin, ${ }^{7}$ alpha-helical, ${ }^{15}$ and random coil,${ }^{14}$ which exhibit different levels of molecular packing. 
Recently, our group has revealed a correlation between the degradation efficiency of a widely utilized enzyme, matrix metalloproteinase-1 (MMP-1), and the $\beta$-sheet content of self-assembled peptide amphiphile (PA) nanofibers. ${ }^{23}$ The MMP-1 cleavability was observed to be significantly reduced in PA nanofibers with stronger $\beta$-sheet content. However, the enzymatic degradation mechanism of these PA nanofibers has not yet been fully elucidated. MMP-1 is an endopeptidase, and therefore it remains unclear whether the MMP-1 acts directly on the nanofibers, disturbing the balance of intramolecular forces (Scheme 1i), or acts on the dissociated monomers, shifting the equilibrium between PA monomers and nanofibers (Scheme 1ii). These processes can consequently lead to erosion, morphological transition or even disassembly of the nanofibers. To test these hypotheses, cathepsin B (CatB) was exploited here to hydrolyze the PA nanofibers. CatB is a lysosomal cysteine protease overexpressed in both invasive and metastatic cancers, ${ }^{24,} 25$ possessing broad specificity for peptide bonds and exhibiting both exopeptidase (carboxypeptidase) and endopeptidase activities. ${ }^{26}$ As such, either cleavage of amino acid residues located on the nanofiber surface, or hydrolysis of cleavable sites buried inside the nanofibers, are likely to occur (Scheme 1). To complement these studies, fluorescence lifetime imaging microscopy (FLIM) combined with the BODIPY-C10 molecular rotor, a fluorophore that is sensitive to the rigidity of its environment, was utilized to directly visualize and quantify the internal viscosity of the PA nanofibers, which may influence their degradation behavior. The findings of this study shed light on how supramolecular peptide nanofibers are degraded by enzymatic hydrolysis, and this knowledge could be applied to develop peptide-based probes for the real-time monitoring of enzymatic activities at disease sites.

Four different PAs were rationally designed in this study (Figure 1A). All the PAs contain a tripeptide head composed of lysine residues (KKK) at the $C$-terminus to display multiple charges 
and ensure good water solubility. The tetrapeptide (GFLG), which has been shown to be preferentially cleaved by CatB and thus widely used to create CatB-responsive materials, was then inserted next to the lysine domain in all the PAs. ${ }^{27,} 28$ Different modifications were implemented at the PA $N$-termini. The well-known $\beta$-sheet forming peptide segment (VVVAAA) was incorporated into PA $1,{ }^{29}$ and an $\alpha$-helix promoting peptide sequence (AAAAAA) was selected for PA2 and PA3, ${ }^{30,31}$ while no structural domain was added into PA4. In addition, compared to PA1, PA2 and PA4, which bear a single palmitic acid tail (C16) at their $N$-termini, PA3 possesses double octanoic acid tails (C8), which has been proven to introduce more steric hindrance and volume to favor a relatively loose molecular packing. ${ }^{32}$ The modifications made in the structural domain and alkyl tail of these PAs are expected to generate assemblies with variable molecular packing.

The designed PAs were synthesized and purified, with their mass and purity confirmed by electrospray ionization mass spectrometry (ESI-MS) and reverse-phase high performance liquid chromatography (RP-HPLC), respectively (Figure S1-4). Owing to their amphiphilic properties, all PAs were observed to self-assemble into long nanofibers (Figure S6) when dissolved in water $(\mathrm{pH} 7)$ at $0.5 \mathrm{mM}$, above their critical aggregation concentrations (CACs) as determined by the Nile red assay (Figure S5). ${ }^{33}$ However, unlike the other three PAs, that retained their long fibrous structure in the enzymatic reaction buffer ( $\mathrm{pH}$ 5), PA4 assemblies appear as short rod-like nanofibers (Figure 1B1-E1), probably due to the increased charge repulsion at $\mathrm{pH} 5$ and lack of structural domain of PA4. Furthermore, incubation in the enzymatic reaction buffer without CatB did not result in degradation or change in the morphology of the PA nanofibers, as observed by HPLC and TEM, respectively (Figure S13 and Figure S14). In the presence of CatB, the fibrous nanostructures observed for PA1, PA2 and PA3 were maintained after a 7 -day incubation at $37^{\circ} \mathrm{C}$, with an increasing degree of erosion observed under TEM (Figure 1B2-D2), while the short rod- 
like nanofibers formed by PA4 had undergone significant degradation, with only irregular aggregates could be observed under TEM (Figure 1E2). To identify the PA fragments after enzymatic hydrolysis by $\mathrm{CatB}$, the reaction solutions were analyzed by liquid chromatography mass spectrometry (LC-MS) at day 7. In addition to the intact PA molecules, PA fragments lacking one $C$-terminal Lys residue were detected from PA1 (Figure S7) and PA2 (Figure S8) reaction solutions. Interestingly, additional PA fragments lacking two Lys residues were detected in both PA3 (Figure S9) and PA4 (Figure S11) solutions, and fragments lacking three Lys residues were also observed for PA4 (Figure S11). Cleavage of the surface lysine residues from PA4 assemblies reduces their solubility in aqueous medium and may lead to their precipitation. The eventual formation of precipitates may explain the absence of defined structures in the TEM images (Figure 1E2). These results demonstrate that CatB can only cleave the $C$-terminal Lys residues presented at the surface of the PA nanofibers residue-by-residue. To validate the endopeptidase activity of CatB through preferential cleavage of the tetra-peptide substrate (GFLG), PA monomers were selected for the hydrolysis experiment. Considering the varying CAC values determined for the different PAs (Figure S5) and the limitations of HPLC detection (UV detection of amide bond) for low peptide concentration, PA3, with the highest CAC among the four PA molecules, was selected as representative PA. The PA3 solution was prepared at $0.05 \mathrm{mM}$, which is below its CAC, and incubated with CatB. The mass spectrum after $24 \mathrm{~h}$ digestion shows that the PA3 monomers have been completely broken down by $\mathrm{CatB}$, since the mass of the original PA3 was not detected. The only PA fragment detected corresponds to the PA cleaved between F and L (Figure S10). The same preferred endopeptidase activity should also be expected for the monomers of the other PA molecules as their structure in the monomer state is very similar and the enzyme would have similar access to the cleavage site in the tetrapeptide (GLFG) substrate. Taken together, these 
results suggest that the activity and selectivity of CatB are influenced by the assembly state of PAs. When the PAs are in their monomeric state, CatB shows predominant endopeptidase activity, preferentially cleaving the peptide bond between F and L. CatB exopeptidase activity may also take place on the PA monomers, but should be at a much slower rate as no related peptide fragments were detected. However, when the PAs are in the assembled state, with the cleavable site buried inside the nanofibers, CatB exopeptidase activity prevails, hydrolyzing surface $C$ terminal amino acid residues sequentially. Therefore, the erosion observed for PA nanofibers resulted from CatB degradation directly on the assembled PA nanofibers. If the CatB cleavage would occur on the dissociated PA monomers, peptide fragments from the endopeptidase cleavage should also be detected. However, even for the PA3 and PA4 nanofibers, which assembled at relatively higher $\mathrm{CAC}$ and with less ordered molecular packing as well as expected higher dissociation rate of the monomers from the nanofibers, no peptide fragments from the endopeptidase cleavage were detected after the 7 days of enzymatic hydrolysis.

The varying degrees of enzymatic degradation observed for the PA nanofibers were further quantified by RP-HPLC (Figure S12). As shown in Figure 2A, the short rod-like PA4 nanofibers exhibited the highest percentage of degradation $(79.07 \pm 2.25 \%)$ after incubation with CatB for 7 days, followed by PA3 and PA2 nanofibers with $53.97 \pm 1.47 \%$ and $36.10 \pm 1.24 \%$ degradation, respectively. By contrast, PA1 nanofibers were barely hydrolysed by CatB with only $5.68 \pm 0.25 \%$ degradation observed after 7 days. The enzymatic degradation efficiency of the PA nanofibers has been found to be negatively correlated to their $\beta$-sheet content in our previous study. ${ }^{23}$ The same correlation was also observed here, as the PA1 nanofibers, with the lowest percentage of degradation, exhibited the strongest $\beta$-sheet signal in the CD spectrum (Figure 2B). Conversely, PA2 nanofibers displayed an $\alpha$-helix secondary structure, and the PA3 and PA4 nanofibers 
exhibited random coil conformations (Figure 2B). The secondary structure of the PA nanofibers, determined by the pattern of hydrogen bonding between the amino hydrogen and carboxyl oxygen atoms in the peptide backbone, reflects the cohesion among the PA molecules in the nanofiber. These cohesive forces can then influence the molecular packing and the stability of the PA nanofibers. The stability of all nanofibers was tested upon 100-fold dilution, by monitoring the emission maximum of the polarity-sensitive Nile red dye. As demonstrated in Figure 2C-D, the emission maximum of Nile red remained at $620 \mathrm{~nm}$ for all nanofibers upon dilution, except for PA4. In the case of PA4 nanofibers, it red-shifted upon dilution, indicating that the nanofibers assembled from PA4 were unstable against dilution, changing the environment of Nile red from low-polarity nanofiber interior to high-polarity aqueous environment. ${ }^{23}$ The low stability of the PA4 nanofibers was consistent with their random coil conformation and the highest degradation efficiency by CatB. To compare the molecular packing in the PA2 and PA3 nanofibers, ThioflavinT (ThT) assay was performed for all the four PAs. ThT was discovered in 1959 as a switch-on fluorescent probe for amyloid fibrils, displaying enhanced fluorescence when bound to $\beta$-sheet rich structures. ${ }^{34}$ It has since been widely used for diagnostic and quantification of amyloid structures, ${ }^{35}$ but also to determine the $\mathrm{CAC}$ of $\beta$-sheet forming peptides ${ }^{36}$ and the $\beta$-sheet content in assembled peptide nanostructures. ${ }^{23,} 32$ The fluorescence on-off status of ThT has been further revealed to be regulated by the change of molecular packing, and could also be induced upon binding to DNA, $\alpha$-helix rich peptide assemblies, polymer membranes, and even sodium dodecyl sulfate (SDS) micelles. ${ }^{37}$ As shown in Figure 2E, the fluorescence intensity of ThT varied significantly when bound to the different PA nanofibers, suggesting different molecular packing in the PA nanofibers: PA1 > PA2 > PA3 > PA4. These results inversely correlate to the degradation 
percentage of PA nanofibers and also the number of $C$-terminal amino acid residues of PA nanofibers being cleaved.

The intermolecular cohesion of these PA nanofibers can also be probed by mapping their internal viscosity, the fluidity of the aliphatic core of the PA nanofibers, which can be visualized and quantified using fluorescence lifetime imaging microscopy (FLIM) combined with a borondipyrromethene (BODIPY) based molecular rotor, BODIPY-C10. ${ }^{38}$ Unlike fluorescence intensity, which changes as a function of fluorophore concentration, the fluorescence lifetime is concentration independent and can provide a quantitative viscosity measurement that is not affected by possible inhomogeneous distribution of the molecular rotor. ${ }^{38,} 39$ The BODIPY-C10 molecular rotor has been extensively exploited to measure the microviscosity of the hydrocarbon region in various lipid-based systems, owing to its lipophilic structure (Figure 3A), which allows its localization in the alkyl chain region of lipid monolayers and bilayers, including biological membranes, without disrupting the lipid structures. ${ }^{40,41}$ Considering the lipophilic core of PA assemblies, BODIPY-C10 was also used here to investigate the internal viscosity of the PA nanofibers. As illustrated in Figure 3B, the PAs were co-assembled with BODIPY-C10 at a molecular ratio of 200:1 to optimise fluorophore signal with self-quenching at high loading (Figure S16a). Upon co-assembly with BODIPY-C10, PA1, PA2 and PA3 were still observed to form nanofibers, which showed no significant differences in both the morphology and secondary structure, compared to those in the absence of the molecular rotor (Figure S15). In comparison, no defined structures were observed for PA4 upon co-assembly, confirming the relatively weak selfassembly ability of PA4 being disturbed in presence of BODIPY-C10 (Figure S15). Once incorporated into the core of PA nanofibers, molecular crowding restricts the intramolecular rotation and suppresses the non-radiative decay of BODIPY-C10, leading to enhanced 
fluorescence quantum yield and lifetime. ${ }^{39}$ As shown in Figure 3C-F, intense emission and long lifetimes were detected from BODIPY-C10 incorporated in the PA nanofibers, indicating that the fluorophore was successfully localized in the alkyl chain core of the PA nanofibers. In contrast, BODIPY-C10 lifetime in an aqueous environment is very short and gives evidence of the dye aggregation. ${ }^{40}$ Additionally, the lifetime of BODIPY-C10 was observed to be independent of the dilution of PA solutions, demonstrating that the fluorophore is not localized externally on the surface of nanofiber bundles (Figure S16b). Diffraction limited fibrous features were imaged for PA1, PA2 and PA3 by FLIM, although single fibres could not be resolved from fibre bundles due to the limiting optical resolution (Figure 3C). The measured fluorescence lifetimes were fitted to a mono-exponential decay model (Figure 3D), which was indicative of the absence of selfquenching or aggregation of the rotors and allowed for the conversion from lifetime to viscosity based on a previously produced calibration curve for BODIPY-C10 (Figure 3F-G). ${ }^{40,}{ }^{41}$ The quantified internal viscosity of the PA nanofibers is in the range 200-800 cP, consistent with liquid phase and equivalent to mixtures of $85-100 \%$ glycerol in methanol at $295 \mathrm{~K}$. It follows the order: $\mathrm{PA} 1=737 \mathrm{cP}>\mathrm{PA} 2=629 \mathrm{cP}>\mathrm{PA} 3=372 \mathrm{cP}$, in agreement with the molecular packing assessed by the ThT assay (Figure 2E). The fluid like range of viscosities measured here for PA1-PA3 nanofibers corresponds to rotational correlation times $(\theta)$ of $c a$. 8-16 ns, measured using timeresolved fluorescence anisotropy measurements (Figure S17), which is similar to values of ca. 620 ns measured previously for the alkyl chain region of related PA nanofibers using EPR spectroscopy. $^{42}$

In conclusion, the enzymatic degradation mechanisms of self-assembled PA nanofibers were revealed through the use of CatB, an enzyme with both endo- and exopeptidase activities. Monomeric PA molecules were preferentially hydrolysed by CatB at a cleavable site located at the 
centre of the molecules. By contrast, assembled PA nanofibers, for which the cleavable site is less accessible, were shown to be degraded by CatB through a surface erosion mechanism whereby external, single amino acids are hydrolyzed in a sequential fashion. Therefore, the observed erosion and disassembly of the PA nanofibers resulted from CatB degradation directly on the assembled nanofibers, rather than on monomers dissociated from the assemblies. Moreover, the internal viscosity of the PA nanofibers was visualized and quantified for the first time at microscopic resolution by FLIM coupled with BODIPY-C10 molecular rotor, a fluorescent probe that increases its lifetime in response to rigidification of its environment. The degradation percentage of the PA nanofibers and the number of cleaved residues from the PA nanofibers were found to be negatively correlated to their internal viscosity. Specifically, high internal viscosity imposed by well-packed $\beta$-sheet conformation of PA molecules was shown to significantly reduce the percentage of degradation and the number of cleaved $C$-terminal amino acid residues. Considering the dynamic nature of this technique, future studies could be performed to measure changes in the internal viscosity of PA nanofibers during enzyme degradation in real time, which may allow the development of peptide-based probes to detect the expression level and activity of diseased-related enzymes. 
Scheme 1 Hypothesized enzymatic degradation mechanisms for the peptide amphiphile (PA) nanofibers. The enzyme can either act (i) directly on the nanofibers, disturbing the balance of intermolecular forces; or (ii) on the dissociated monomers, shifting the equilibrium between PA monomers and nanofibers. These hydrolysis processes can consequently lead to erosion, morphological transition or even disassembly of the nanofibers.

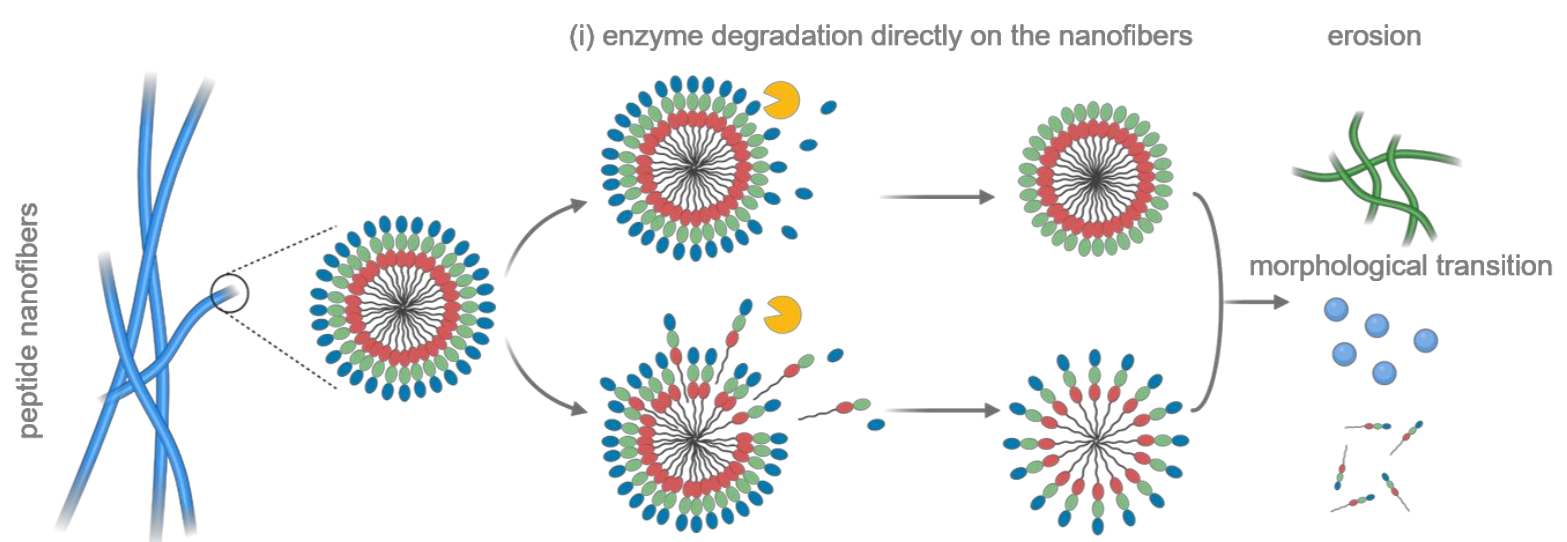

(ii) enzyme degradation on the dissociated monomers

disassembly 
2

3

4

5

6

7

8

9

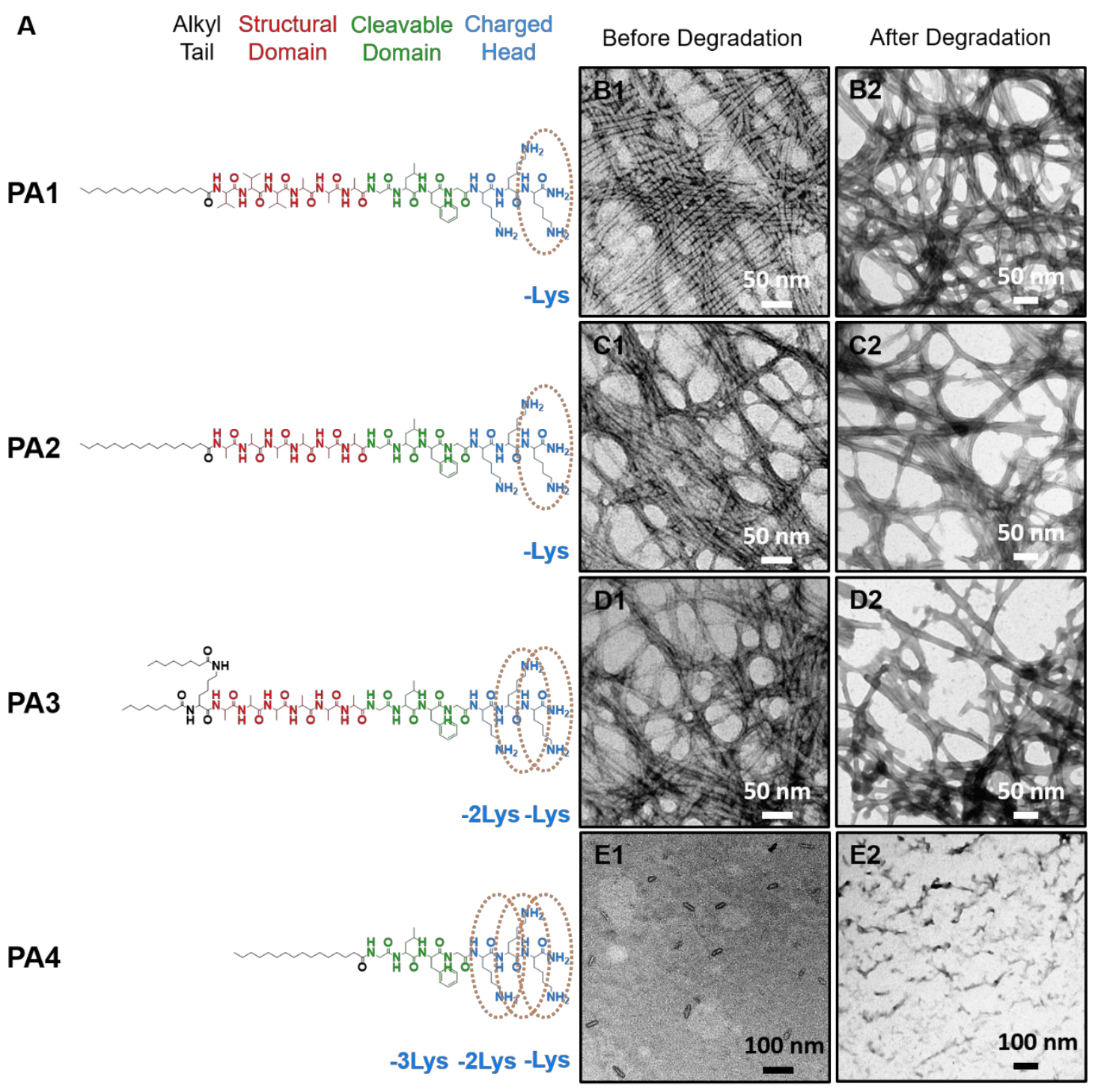

Figure 1. Molecular design, assembly and CatB degradation of peptide amphiphile (PA) nanofibers. (A) Chemical structures of the rationally designed PAs containing four key design elements: (i) hydrophobic alkyl tail (black); (ii) secondary structure forming domain (red); (iii) CatB cleavable domain (green) and (iv) hydrophilic peptide headgroup (blue); together with circular labels (orange) demonstrating the $C$-terminal amino acid residue(s) cleaved by CatB in $168 \mathrm{~h}$. Representative TEM images of the PA assemblies in enzymatic reaction buffer (pH 5) before (B1E1) and after (B2-E2) degradation by CatB for 168 h: (B) PA1; (C) PA2; (D) PA3; (E) PA4. 

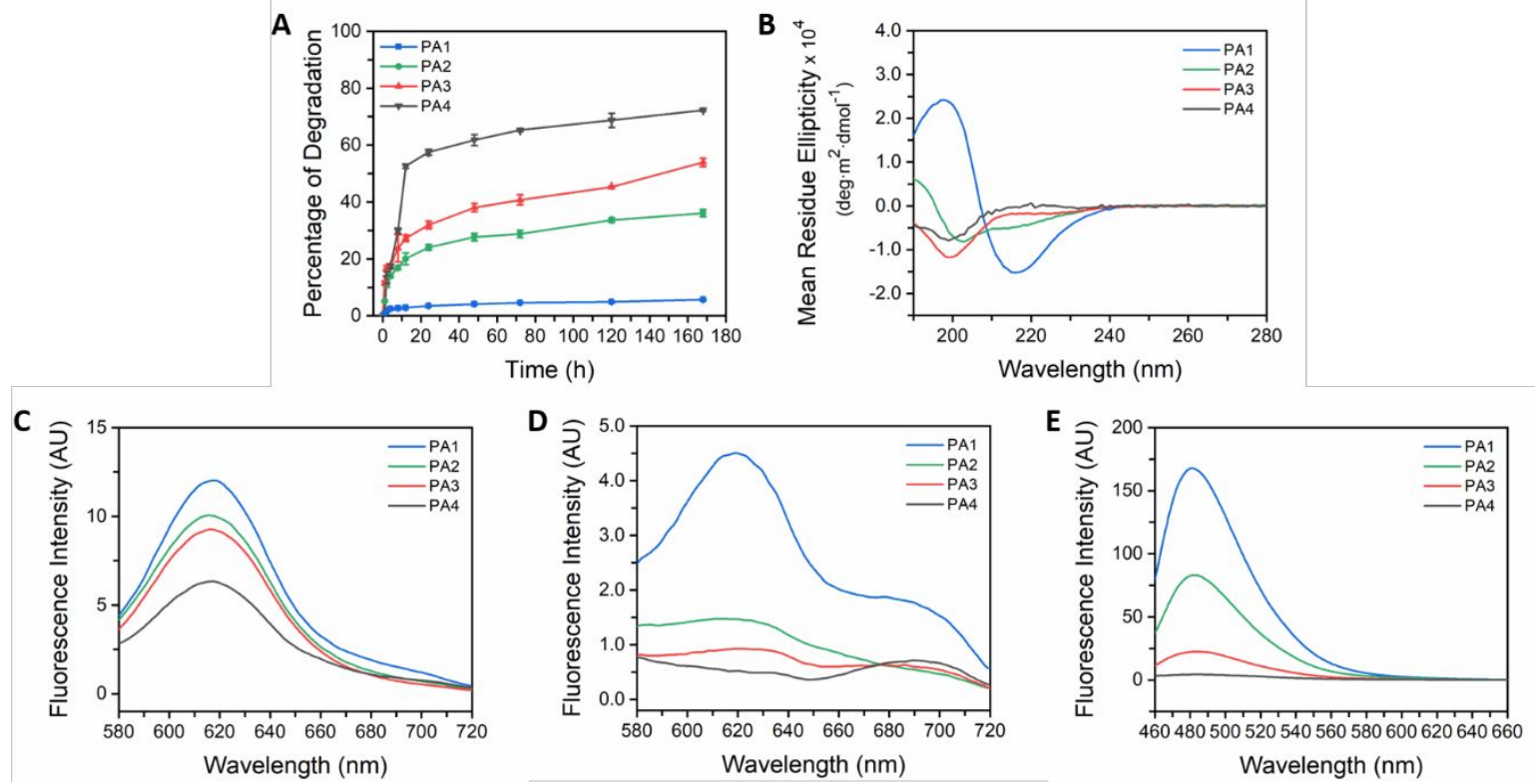

Figure 2. Cathepsin B (CatB) degradation profile and structure characterization of peptide amphiphile (PA) nanofibers. (A) Degradation profile of the PA nanofibers by CatB during a 7-day (168 h) period, monitored and quantified over time using reverse-phase high performance liquid chromatography (RP-HPLC). (B) CD specta showing $\beta$-sheet secondary structure for PA1 nanofibers, $\alpha$-helix for PA2 nanofibers, and random coil conformation for PA3 and PA4 nanofibers. (C) Fluorescence spectra of Nile red $\left(\lambda_{\mathrm{ex}}=550 \mathrm{~nm}\right)$ in the presence of $0.5 \mathrm{mM}$ PAs showing emission maximum around wavelength of $620 \mathrm{~nm}$, indicative of PA aggregation. (D) Upon 100-fold dilution, the emission maximum of Nile red remained around wavelength of $620 \mathrm{~nm}$ for PA1, PA2 and PA3, indicating formation of stable nanostructures by these PAs (Supplementary Discussion S4); while the emission maximum of the dye red-shiftted for PA4, showing that the formed nanostructures were unstable. (E) Fluorescence spectra of ThT $\left(\lambda_{\text {ex }}\right.$ $=440 \mathrm{~nm}$ ) in the presence of PA nanofibers indicating differences in the molecular packing of the various PA nanofibers (PA1 $>$ PA2 $>$ PA3 $>$ PA4). 
A
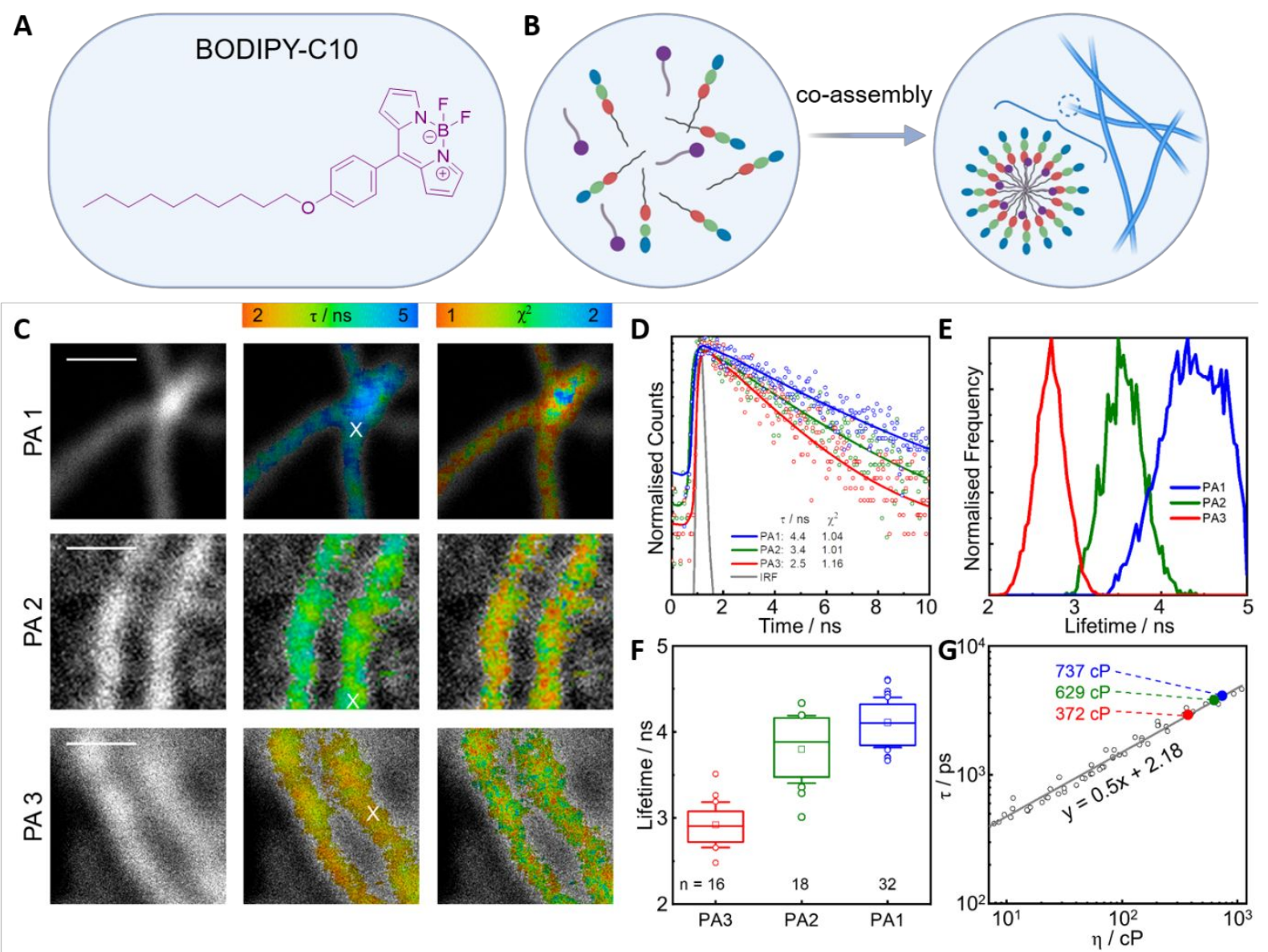

Figure 3. Internal viscosity of peptide amphiphile (PA) nanofibers measured by Fluorescence Lifetime Imaging Microscopy (FLIM) and using BODIPY-C10 molecular rotor. (A) Chemical structure of BODIPY-C10 molecular rotor. (B) Proposed co-assembly of the BODIPY-C10 with PA molecules into nanofibers. (C) FLIM maps of PA1, PA2 and PA3 nanofibers in solution. Higher lifetime is indicated by blue colour in the FLIM maps, which corresponds to higher viscosity. (D) Typical time-resolved fluorescence decay traces taken from the crosshairs in (C). (E) Fluorescence lifetime histograms recorded from the images in (C). (F) Box plot of average nanofiber lifetimes, calculated from the peak of histogram distribution within a single image. Mean lifetime in ns are calculated as $(95 \%$ confidence interval): PA1 = 4.11 (4.00 - 4.21), PA2 = 3.80 (3.60 - 3.99), and PA3 = $2.92(2.78-3.06)$. (G) BODIPYC10 calibration between lifetime and viscosity, measured in methanol/glycerol mixtures of varied composition (0$100 \%$ ) over a 278 to $334 \mathrm{~K}$ temperature range, adapted from reference ${ }^{40,41}$ Coloured dots are the mean lifetimes of all nanofibers measured in $(\mathbf{F})$, labelled with the corresponding viscosity. Scale bars $=1 \mu \mathrm{m}$. 


\begin{abstract}
ASSOCIATED CONTENT
Supporting Information. Detailed description of experimental materials and methods; supplementary figures showing characterization of PA molecules (RP-HPLC, ESI-MS and CAC); analysis and identification of the cleaved PA fragments after incubation with CatB (RP-HPLC and ESI-MS); characterization of BODIPY-C10/PA co-assemblies (TEM and CD) (PDF).
\end{abstract}

\title{
AUTHOR INFORMATION \\ Corresponding Authors \\ *(H.S.A.) E-mail: $\underline{\text { h.azevedo@qmul.ac.uk }}$ \\ *(M.K.K.) E-mail: m.kuimova@imperial.ac.uk \\ Author Contributions
}

Y.S. conceptualized, designed, and performed experiments, analyzed data, and wrote the manuscript. P.A.S. performed fluorescence lifetime imaging experiments, analyzed these data, and wrote the manuscript. M.K.K and H.S.A. supervised the research and edited the manuscript. All authors have given approval to the final version of the manuscript. $\$$ These authors contributed equally.

\section{Notes}

The authors declare no competing financial interest. 


\section{ACKNOWLEDGMENT}

Y.S. and H.S.A. gratefully acknowledge the Seed Award in Science (210122/Z/18/Z) granted by the Wellcome Trust. M.K.K. is grateful to the EPSRC for Career Acceleration Fellowship (EP/I003983/1). P.A.S. and M. K. K acknowledge the funding from the Excellence Fund for Frontier Research, Imperial College London.

\section{REFERENCES}

(1) Zelzer, M.; Todd, S. J.; Hirst, A. R.; McDonald, T. O.; Ulijn, R. V., Enzyme responsive materials: design strategies and future developments. Biomaterials science 2013, 1 (1), 11-39. (2) Anderson, C. F.; Cui, H., Protease-Sensitive Nanomaterials for Cancer Therapeutics and Imaging. Industrial \& engineering chemistry research 2017, 56 (20), 5761-5777.

(3) Mu, J.; Lin, J.; Huang, P.; Chen, X., Development of endogenous enzyme-responsive nanomaterials for theranostics. Chemical Society reviews 2018, 47 (15), 5554-5573.

(4) Jun, H. W.; Yuwono, V.; Paramonov, S. E.; Hartgerink, J. D., Enzyme - mediated degradation of peptide - amphiphile nanofiber networks. Adv Mater 2005, 17 (21), 2612-2617.

(5) Chau, Y.; Luo, Y.; Cheung, A. C.; Nagai, Y.; Zhang, S.; Kobler, J. B.; Zeitels, S. M.; Langer, R., Incorporation of a matrix metalloproteinase-sensitive substrate into self-assembling peptides a model for biofunctional scaffolds. Biomaterials 2008, 29 (11), 1713-9.

(6) Galler, K. M.; Aulisa, L.; Regan, K. R.; D'Souza, R. N.; Hartgerink, J. D., Self-assembling multidomain peptide hydrogels: designed susceptibility to enzymatic cleavage allows enhanced cell migration and spreading. Journal of the American Chemical Society 2010, 132 (9), 3217-23. (7) Giano, M. C.; Pochan, D. J.; Schneider, J. P., Controlled biodegradation of self-assembling beta-hairpin peptide hydrogels by proteolysis with matrix metalloproteinase-13. Biomaterials 2011, 32 (27), 6471-7.

(8) Yang, Z.; Ma, M.; Xu, B., Using matrix metalloprotease-9 (MMP-9) to trigger supramolecular hydrogelation. Soft Matter 2009, 5 (13), 2546-2548.

(9) Gao, Y.; Shi, J.; Yuan, D.; Xu, B., Imaging enzyme-triggered self-assembly of small molecules inside live cells. Nature communications 2012, 3, 1033.

(10) Hirst, A. R.; Roy, S.; Arora, M.; Das, A. K.; Hodson, N.; Murray, P.; Marshall, S.; Javid, N.; Sefcik, J.; Boekhoven, J.; van Esch, J. H.; Santabarbara, S.; Hunt, N. T.; Ulijn, R. V., Biocatalytic induction of supramolecular order. Nature chemistry 2010, 2 (12), 1089-94.

(11) Webber, M. J.; Newcomb, C. J.; Bitton, R.; Stupp, S. I., Switching of Self-Assembly in a Peptide Nanostructure with a Specific Enzyme. Soft Matter 2011, 7 (20), 9665-9672.

(12) Koda, D.; Maruyama, T.; Minakuchi, N.; Nakashima, K.; Goto, M., Proteinase-mediated drastic morphological change of peptide-amphiphile to induce supramolecular hydrogelation. Chemical communications 2010, 46 (6), 979-81. 
(13) Dehsorkhi, A.; Hamley, I. W.; Seitsonen, J.; Ruokolainen, J., Tuning self-assembled nanostructures through enzymatic degradation of a peptide amphiphile. Langmuir 2013, 29 (22), 6665-72.

(14) Kalafatovic, D.; Nobis, M.; Javid, N.; Frederix, P. W.; Anderson, K. I.; Saunders, B. R.; Ulijn, R. V., MMP-9 triggered micelle-to-fibre transitions for slow release of doxorubicin. Biomaterials science 2015, 3 (2), 246-9.

(15) Shi, Y.; Hu, Y.; Ochbaum, G.; Lin, R.; Bitton, R.; Cui, H.; Azevedo, H. S., Enzymatic activation of cell-penetrating peptides in self-assembled nanostructures triggers fibre-to-micelle morphological transition. Chemical communications 2017, 53 (52), 7037-7040.

(16) Tanaka, A.; Fukuoka, Y.; Morimoto, Y.; Honjo, T.; Koda, D.; Goto, M.; Maruyama, T., Cancer cell death induced by the intracellular self-assembly of an enzyme-responsive supramolecular gelator. Journal of the American Chemical Society 2015, 137 (2), 770-5.

(17) Zhou, J.; Du, X.; Yamagata, N.; Xu, B., Enzyme-Instructed Self-Assembly of Small DPeptides as a Multiple-Step Process for Selectively Killing Cancer Cells. Journal of the American Chemical Society 2016, 138 (11), 3813-23.

(18) Son, J.; Kalafatovic, D.; Kumar, M.; Yoo, B.; Cornejo, M. A.; Contel, M.; Ulijn, R. V., Customizing Morphology, Size, and Response Kinetics of Matrix Metalloproteinase-Responsive Nanostructures by Systematic Peptide Design. ACS nano 2019, 13 (2), 1555-1562.

(19) Kim, J. K.; Anderson, J.; Jun, H. W.; Repka, M. A.; Jo, S., Self-assembling peptide amphiphile-based nanofiber gel for bioresponsive cisplatin delivery. Molecular pharmaceutics 2009, 6 (3), 978-85.

(20) Kalafatovic, D.; Nobis, M.; Son, J.; Anderson, K. I.; Ulijn, R. V., MMP-9 triggered selfassembly of doxorubicin nanofiber depots halts tumor growth. Biomaterials 2016, 98, 192-202. (21) Chakroun, R. W.; Sneider, A.; Anderson, C. F.; Wang, F.; Wu, P. H.; Wirtz, D.; Cui, H., Supramolecular Design of Unsymmetric Reverse Bolaamphiphiles for Cell-Sensitive Hydrogel Degradation and Drug Release. Angewandte Chemie 2020, 59 (11), 4434-4442.

(22) Ferreira, D. S.; Lin, Y. A.; Cui, H.; Hubbell, J. A.; Reis, R. L.; Azevedo, H. S., Molecularly engineered self-assembling membranes for cell-mediated degradation. Advanced healthcare materials 2015, 4 (4), 602-12.

(23) Shi, Y.; Ferreira, D. S.; Banerjee, J.; Pickford, A. R.; Azevedo, H. S., Tuning the matrix metalloproteinase-1 degradability of peptide amphiphile nanofibers through supramolecular engineering. Biomaterials science 2019, 7 (12), 5132-5142.

(24) Aggarwal, N.; Sloane, B. F., Cathepsin B: multiple roles in cancer. Proteomics. Clinical applications 2014, 8 (5-6), 427-37.

(25) Olson, O. C.; Joyce, J. A., Cysteine cathepsin proteases: regulators of cancer progression and therapeutic response. Nature reviews. Cancer 2015, 15 (12), 712-29.

(26) Turk, V.; Stoka, V.; Vasiljeva, O.; Renko, M.; Sun, T.; Turk, B.; Turk, D., Cysteine cathepsins: from structure, function and regulation to new frontiers. Biochimica et biophysica acta 2012, 1824 (1), 68-88.

(27) Rejmanová, P.; Pohl, J.; Baudyš, M.; Kostka, V.; Kopeček, J., Degradation of oligopeptide sequences in N-(2-hydroxypropyl) methacrylamide copolymers by bovine spleen cathepsin B. Makromol Chem 1983, 184, 2009-2020.

(28) Lock, L. L.; Cheetham, A. G.; Zhang, P.; Cui, H., Design and construction of supramolecular nanobeacons for enzyme detection. ACS nano 2013, 7 (6), 4924-32. 
(29) Cui, H.; Webber, M. J.; Stupp, S. I., Self-assembly of peptide amphiphiles: from molecules to nanostructures to biomaterials. Biopolymers 2010, 94 (1), 1-18.

(30) Levitt, M., Conformational preferences of amino acids in globular proteins. Biochemistry 1978, 17 (20), 4277-85.

(31) Radvar, E.; Shi, Y.; Grasso, S.; Edwards-Gayle, C. J. C.; Liu, X.; Mauter, M. S.; Castelletto, V.; Hamley, I. W.; Reece, M. J.; H, S. A., Magnetic Field-Induced Alignment of Nanofibrous Supramolecular Membranes: A Molecular Design Approach to Create Tissue-like Biomaterials. ACS applied materials \& interfaces 2020, 12 (20), 22661-22672.

(32) Li, Y.; Wang, Y.; Ou, S. H.; Lock, L. L.; Xu, X.; Ghose, S.; Li, Z. J.; Cui, H., Conformation Preservation of alpha-Helical Peptides within Supramolecular Filamentous Assemblies.

Biomacromolecules 2017, 18 (11), 3611-3620.

(33) Shi, Y.; Lin, R.; Cui, H.; Azevedo, H. S., Multifunctional Self-Assembling Peptide-Based Nanostructures for Targeted Intracellular Delivery: Design, Physicochemical Characterization, and Biological Assessment. Methods in molecular biology 2018, 1758, 11-26.

(34) Vassar, P. S.; Culling, C. F., Fluorescent stains, with special reference to amyloid and connective tissues. Archives of pathology 1959, 68, 487-98.

(35) Biancalana, M.; Koide, S., Molecular mechanism of Thioflavin-T binding to amyloid fibrils. Biochimica et biophysica acta 2010, 1804 (7), 1405-12.

(36) Edwards-Gayle, C. J. C.; Barrett, G.; Roy, S.; Castelletto, V.; Seitsonen, J.; Ruokolainen, J.; Hamley, I. W., Selective Antibacterial Activity and Lipid Membrane Interactions of ArginineRich Amphiphilic Peptides. ACS applied bio materials 2020, 3 (2), 1165-1175.

(37) Amdursky, N.; Erez, Y.; Huppert, D., Molecular rotors: what lies behind the high sensitivity of the thioflavin-T fluorescent marker. Accounts of chemical research 2012, 45 (9), 1548-57.

(38) Vyšniauskas, A.; Kuimova, M. K., A twisted tale: measuring viscosity and temperature of microenvironments using molecular rotors. International Reviews in Physical Chemistry 2018, 37 (2), 259-285.

(39) Kuimova, M. K., Mapping viscosity in cells using molecular rotors. Physical Chemistry Chemical Physics 2012, 14 (37), 12671-12686.

(40) Hosny, N. A.; Mohamedi, G.; Rademeyer, P.; Owen, J.; Wu, Y.; Tang, M. X.; Eckersley, R. J.; Stride, E.; Kuimova, M. K., Mapping microbubble viscosity using fluorescence lifetime imaging of molecular rotors. Proceedings of the National Academy of Sciences of the United States of America 2013, 110 (23), 9225-30.

(41) Wu, Y.; Stefl, M.; Olzynska, A.; Hof, M.; Yahioglu, G.; Yip, P.; Casey, D. R.; Ces, O.; Humpolickova, J.; Kuimova, M. K., Molecular rheometry: direct determination of viscosity in Lo and Ld lipid phases via fluorescence lifetime imaging. Physical chemistry chemical physics : PCCP 2013, 15 (36), 14986-93.

(42) Ortony, J. H.; Newcomb, C. J.; Matson, J. B.; Palmer, L. C.; Doan, P. E.; Hoffman, B. M.; Stupp, S. I., Internal dynamics of a supramolecular nanofibre. Nature materials 2014, 13 (8), 812-6. 


\section{For Table of Contents Only}

\section{Enzymatic Degradation Mechanism}

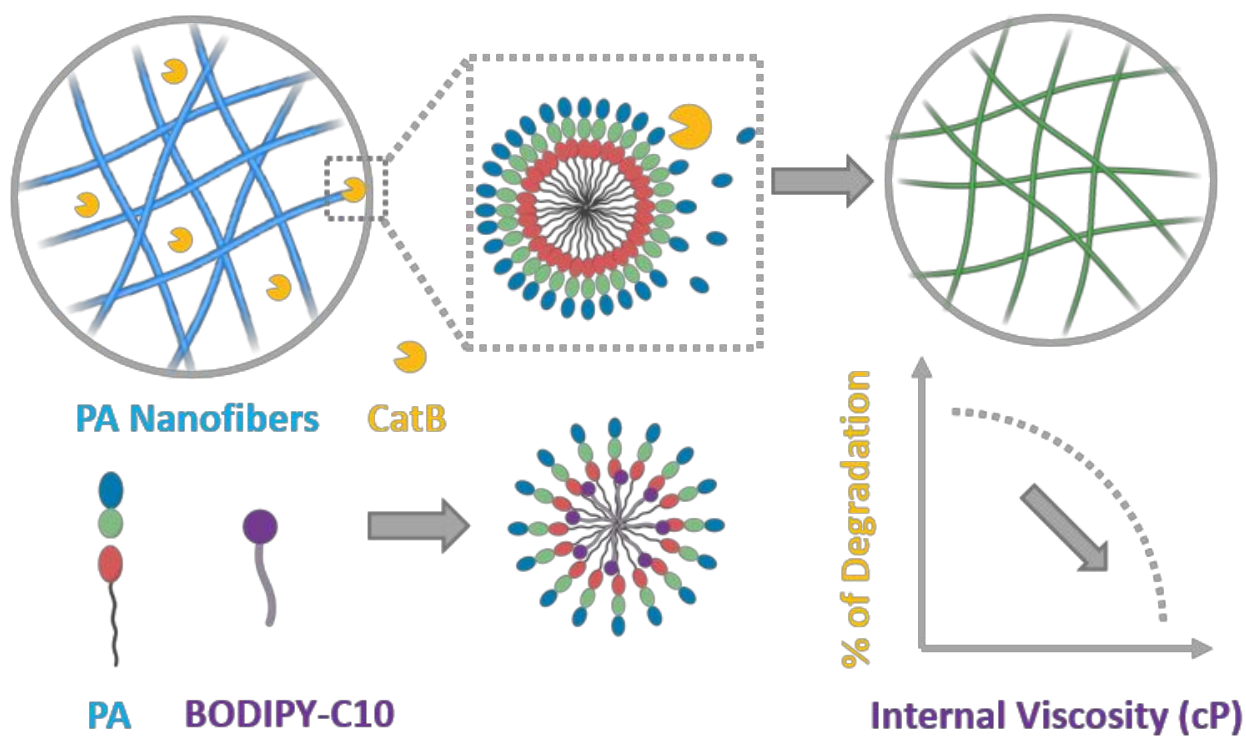

